\title{
Fluctuation conductivity and microscopic granularity in Bi-based high-temperature superconductors
}

\author{
A. R. Jurelo, J. V. Kunzler, J. Schaf, and P. Pureur* \\ Instituto de Física, Universidade Federal do Rio Grande do Sul, P.O. Box 15051, 91501-970 Porto Alegre, Brazil \\ J. Rosenblatt \\ Laboratoire de Physique des Solides, ERS F0134 au CNRS, INSA, B.P. 14A, F-35043 Rennes Cedex, France
}

(Received 24 July 1997)

\begin{abstract}
Systematic conductivity measurements near $T_{c}$ in granular $(\mathrm{Bi}, \mathrm{Pb})-\mathrm{Sr}-\mathrm{Ca}-\mathrm{Cu}-\mathrm{O}$ superconductors are presented, and focus is given on the interplay between thermal fluctuations and disorder at micro- and mesoscopic levels. Experiments show that the resistive transition is a two-step process. In the normal phase, Gaussian and critical fluctuation conductivity regimes were identified. Both are affected by local disorder. Particularly, the critical regime is characterized by a power law with exponent $\lambda_{\mathrm{cr}} \sim 3$, which we interpret as resulting from microscopic granularity. Below $T_{c}$ and in the regime describing the approach to the zero-resistance state, fluctuation conductivity diverges as expected in a paracoherent-coherent transition of a mesoscopic granular superconductor. The results show that, instead of trivially rounding the transition, disorder at micro- and mesoscopic levels preserves a true critical phenomenology in granular superconductors.
\end{abstract}

[S0163-1829(97)06245-0]

\section{INTRODUCTION}

High- $T_{c}$ superconductors are known to show large regions dominated by thermal fluctuations. Most specimens of these materials are also known to present strong structural disorder at microscopic and mesoscopic levels. However, only a few studies were devoted to investigating the interplay between these two important aspects of the high- $T_{c}$ systems. Mostly, it has been argued that inhomogeneities producing a nonuniform transition temperature lead to noncritical roundings in the equilibrium ${ }^{1}$ and transport $^{2}$ properties near $T_{c}$. Although this approach is likely to be correct in the limit of small dispersion in $T_{c}$, the situation is less clear in the case of strong disorder.

Recently, Rosenblatt et al..$^{3,4}$ have introduced the concept of microscopic intragrain granularity to explain the scalinglike behavior of the field of first flux penetration into polycrystalline samples of fluorine-doped $\mathrm{YBa}_{2} \mathrm{Cu}_{3} \mathrm{O}_{7-\delta}$. These authors argue that in the superconducting cuprates, due to the smallness of the coherence length, an unavoidable coupling is produced between microscopic defects (e.g., deviations of the stoichiometry) and spatial fluctuations of the critical temperature, $T_{c}(\mathbf{r})$. A local variation of the critical temperature is conceivable if inhomogeneities extend over distances of the order or larger than the intrinsic superconducting coherence length $\xi(T)$, making thus quite plausible the occurrence of inhomogeneous superconductivity in certain samples of the high- $T_{c}$ systems. According to the model in Ref. 3, there are two related conditions for a supercurrent to freely circulate between positions $\mathbf{r}$ and $\mathbf{r}^{\prime}$ of a microscopically disordered superconductor: (i) $\mathbf{r}$ and $\mathbf{r}^{\prime}$ must belong to the same single connected region, or cluster, defined by $T_{c}(\mathbf{r})>T$, where $T$ is the temperature, and (ii) the order-parameter correlation function $\left\langle\psi(\mathbf{r}) \psi\left(\mathbf{r}^{\prime}\right)\right\rangle$ should be nonzero within the cluster, that is $\left|\mathbf{r}-\mathbf{r}^{\prime}\right| \leqslant \xi(T)$. Condition (i) defines a temperature-dependent percolation parameter such that for $p\left(T_{c}\right)=p_{c}$ an infinite cluster spans the sample and infinite conductivity should be obtained. Condition (ii) means that the characteristic percolation correlation length in the system is given approximately by $\xi(T)$, which implies that $p(T)$ is independent of the particular distribution of $T_{c}(\mathbf{r})$. The model of Ref. 3 predicts that the critical exponent for the correlation length of this temperature controlled percolation problem is $v \cong \frac{4}{3}$, i.e., the same value found in genuine granular material. ${ }^{5}$ In this sense microscopic disorder and granularity are equivalent. An important consequence of this approach is that, instead of blurring the transition, the microscopic granularity model preserves a true critical phenomenology, where physical quantities behave as power laws with the same exponents characterizing the superconducting transition in mesoscopic granular networks. These exponents are known to be large. ${ }^{5}$ This implies that in some circumstances it may be difficult to clearly distinguish between a genuine power-law behavior and a mere noncritical rounding.

In this paper, we report on fluctuation conductivity measurements in polycrystalline $\mathrm{Bi}_{1.6} \mathrm{~Pb}_{0.4} \mathrm{Sr}_{2} \mathrm{CaCu}_{2} \mathrm{O}_{8+x}$ and $\mathrm{Bi}_{1.6} \mathrm{~Pb}_{0.4} \mathrm{Sr}_{2} \mathrm{Ca}_{2} \mathrm{Cu}_{3} \mathrm{O}_{10+y}$. We analyze the results with the logarithmic temperature derivative of the conductivity. ${ }^{6}$ The method is particularly appropriate to identify power-law divergences of the conductivity and does not require fitting procedures involving adjustable parameters. Above, but close to $T_{c}$, we have clearly and systematically found that the fluctuation conductivity is described by power-law regimes with exponent $\lambda_{\mathrm{cr}} \sim 3$, which we identify with microscopic granularity. Indeed, this exponent is long known to characterize the critical resistive transition in "classical" granular arrays formed by metallic superconducting particles embedded in a poorly conducting matrix. ${ }^{5}$ Our samples, however, are loosely packed ceramic systems, where the 
TABLE I. Exponents that characterize conductivity fluctuations in polycrystalline samples of $\mathrm{Bi}_{1.6} \mathrm{~Pb}_{0.4} \mathrm{Sr}_{2} \mathrm{CaCu}_{2} \mathrm{O}_{8+x}(\mathrm{Bi}-2212)$ and $\mathrm{Bi}_{1.6} \mathrm{~Pb}_{0.4} \mathrm{Sr}_{2} \mathrm{Ca}_{2} \mathrm{Cu}_{3} \mathrm{O}_{10+x}$ (Bi-2223). Fluctuations in the normal phase $\left(T>T_{c}\right)$ correspond to Gaussian $\left(\lambda_{G}\right)$ and critical $\left(\lambda_{\mathrm{cr}}\right)$ exponents. In the paracoherent state that describes the approach to the $R=0$ state $\left(T<T_{c}\right)$ the exponents are $s$ and $s^{\prime}$. The temperature $T_{p}$ indicates the position of the maximum in $d \rho / d T$ and is useful to delimit the temperature intervals relevant for the analysis of conductivity fluctuations in the normal and paracoherent states. The reported uncertainties in the exponents result from averages over the $N$ measurements of the resistive transition performed per sample. The heat treatment of each sample is also indicated. The standard procedure described in the text is denoted by as-sint (as-sintered). Additional annealings are indicated by the temperature and time duration.

\begin{tabular}{|c|c|c|c|c|c|c|c|}
\hline \multirow[b]{2}{*}{ Sample } & \multirow[b]{2}{*}{ Heat treatment } & \multicolumn{3}{|c|}{$T<T_{c}$} & \multirow[b]{2}{*}{$T_{p}(\mathrm{~K})$} & \multicolumn{2}{|c|}{$T>T_{c}$} \\
\hline & & $N$ & $s$ & $s^{\prime}$ & & $\lambda_{\mathrm{cr}}$ & $\lambda_{G}$ \\
\hline \multicolumn{8}{|l|}{ Bi-2212 } \\
\hline B2-a & as-sint. & 7 & $3.0 \pm 0.2$ & $4.3 \pm 0.1$ & 73.3 & $3.0 \pm 0.1$ & $0.50 \pm 0.05$ \\
\hline B2-b & $250{ }^{\circ} \mathrm{C}-12 \mathrm{~h}$ & 3 & $3.1 \pm 0.5$ & & 64.4 & $3.0 \pm 0.3$ & $0.47 \pm 0.05$ \\
\hline B2-c & $650{ }^{\circ} \mathrm{C}-24 \mathrm{~h}$ & 5 & $3.1 \pm 0.1$ & $4.4 \pm 0.4$ & 78.4 & & $0.51 \pm 0.03$ \\
\hline \multicolumn{8}{|l|}{ Bi-2223 } \\
\hline B3-I & as-sint. & 12 & $3.0 \pm 0.4$ & & 109.9 & $3.1 \pm 0.3$ & $0.84 \pm 0.13$ \\
\hline B3-II & $270{ }^{\circ} \mathrm{C}-3 \mathrm{~h}$ & 4 & $3.1 \pm 0.3$ & & 108.6 & $3.2 \pm 0.1$ & $0.86 \pm 0.16$ \\
\hline B3-III & $650^{\circ} \mathrm{C}-3 \mathrm{~h}$ & 9 & & $4.1 \pm 0.2$ & 109.1 & $2.8 \pm 0.4$ & $1.1 \pm 0.20$ \\
\hline B3-IV & $650^{\circ} \mathrm{C}-12 \mathrm{~h}$ & 9 & $2.9 \pm 0.1$ & & 108.9 & & $0.55 \pm 0.10$ \\
\hline
\end{tabular}

sizes of crystallites vary between 0.1 and $20 \mu \mathrm{m}$. This means that these specimens are representative of genuine mesoscopic granular superconductors, where infinite conductivity is achieved through activation of weak links between the crystal grains. Thus, in addition to effects of the intracrystallite microscopic granularity, whose fluctuation regimes are observed in the normal phase, we have also studied the regimes describing the approach to the zero resistance state. We have indeed obtained that these regimes are dominated by thermal fluctuations typical of mesoscopic granular arrays, as previously found in ceramic $\mathrm{YBa}_{2} \mathrm{Cu}_{3} \mathrm{O}_{7-\delta}$ (Refs. 6 and 7), and are characteristic of a paracoherent-coherent transition. ${ }^{5}$ In most of the measurements, the effects of microscopic granularity could be clearly separated from those resulting from "classical" mesoscopic granularity.

\section{EXPERIMENT}

Three polycrystalline samples of composition $\mathrm{Bi}_{1.6} \mathrm{~Pb}_{0.4} \mathrm{Sr}_{2} \mathrm{CaCu}_{2} \mathrm{O}_{8+x}$ (Bi-2212) and four of $\mathrm{Bi}_{1.6} \mathrm{~Pb}_{0.4} \mathrm{Sr}_{2} \mathrm{Ca}_{2} \mathrm{Cu}_{3} \mathrm{O}_{10+y}$ (Bi-2223) were prepared from the precursors $\mathrm{Bi}_{2} \mathrm{O}_{3}, \mathrm{PbO}, \mathrm{CuO}, \mathrm{SrCO}_{3}$, and $\mathrm{CaCO}_{3}$. The preparation of these compounds proceeds in three stages. First, calcination of the mixed and ground precursors was performed in air at $800{ }^{\circ} \mathrm{C}$, for $20 \mathrm{~h}$. After a slow decrease of the temperature, the samples were ground, pressed into pellets, and annealed for $20 \mathrm{~h}$ at $820^{\circ} \mathrm{C}$ or $825^{\circ} \mathrm{C}$, for Bi-2212 or Bi-2223, respectively. After another grinding, the final $160 \mathrm{~h}$ sintering was done at $800{ }^{\circ} \mathrm{C}$ for $\mathrm{Bi}-2212$, and $850{ }^{\circ} \mathrm{C}$ for $\mathrm{Bi}-2223$. Some of the samples were submitted to further annealing in order to generate a variety of states of mesoscopic and microscopic disorder. For instance, one of the Bi2212 and one of the $\mathrm{Bi}-2223$ specimens were annealed at $650{ }^{\circ} \mathrm{C}$ for $12 \mathrm{~h}$ in flowing oxygen. This procedure removes some of the oxygen excess in the grains, but leads to precipitation of $\mathrm{Ca}_{2} \mathrm{PbO}_{4}$ at the grain frontiers ${ }^{8}$ and strongly enhances the effects of mesoscopic granularity in the resistive transition. In Table I, which lists the prepared samples, is indicated the heat treatment performed in addition to the assintered state.
The resistivity measurements were performed using a low-frequency ac technique. Four electrical contacts were obtained by sputtering thick silver films in the form of strips on the surface of bar-shaped samples. Copper wires were then attached to the contacts with silver paint. After a cure at $300{ }^{\circ} \mathrm{C}$, contact resistances less than $0.5 \Omega$ could be obtained. Relative sensitivities of $10^{-5}$ were attained in the resistivity measurements. Current densities ranging from 3 to $800 \mathrm{~mA} / \mathrm{cm}^{2}$ were employed. Temperatures were determined with a Pt sensor with an accuracy of 1-2 $\mathrm{mK}$. The data points were recorded while increasing or decreasing the temperature in sweeping rates of $3 \mathrm{~K} / \mathrm{h}$ near the transition. A large number of closely spaced points was collected in order to allow for a numerical calculation of the temperature derivative of the resistivity, $d \rho / d T$, in the vicinity of $T_{c}$.

\section{METHOD OF ANALYSIS}

We analyze our data by adopting the simplest scaling approach for the fluctuation conductivity, i.e.,

$$
\Delta \sigma=A\left(T-T_{c}\right)^{-\lambda},
$$

where $\lambda$ is the critical exponent, $A$ is a constant and $\Delta \sigma$ $=\sigma-\sigma_{R}$ is obtained from the measured conductivity $(\sigma)$ by subtracting the regular contribution, $\sigma_{R}$. As usual, $\sigma_{R}$ is estimated by extrapolating the high-temperature behavior

$$
\sigma \cong \sigma_{R}=\frac{1}{a+b T}
$$

to the vicinity of $T_{c}$. In Eq. (2) $a$ and $b$ are constants. The linear resistivity behavior is verified above $200 \mathrm{~K}$ in both systems.

We determine numerically the logarithmic temperature derivative of $\Delta \sigma$, and define

$$
\chi_{\sigma}=-\frac{d}{d T} \ln (\Delta \sigma)
$$

Then, using Eq. (1) we obtain

$$
\frac{1}{\chi_{\sigma}}=\frac{1}{\lambda}\left(T-T_{c}\right) .
$$


The quantity $\chi_{\sigma}$ is formally analogous to a Curie-Weiss susceptibility in a ferromagnet, and the critical exponent plays the role of the Curie constant. Thus, simple identification of linear temperature behavior in plots of $1 / \chi_{\sigma}$ vs $T$ allows the simultaneous determination of $T_{c}$ and $\lambda$. The amplitude $A$ remains undetermined, although it can be retrieved with the help of Eq. (1) once $T_{c}$ and $\lambda$ are obtained.

The main source of uncertainty in our method comes from the extrapolation procedure to estimate $\sigma_{R}$ near $T_{c}$. Numerical calculation of $d(\Delta \sigma) / d T$ also introduces errors, but these tend to be relatively less significant close to $T_{c}$, where $d \rho / d T$ is large. Nevertheless, in order to improve the reliability of the method used we repeated a given experiment several times. This allows us to judge about the quality of the fits and extract the values of the relevant parameters on the basis of an average set of measurements. When repeating an experiment, some conditions may be varied, like increasing or decreasing the temperature or changing the intensity of the measuring current. The number of experiments per sample is indicated in Table I.

To illustrate our strategy, in Fig. 1 we show a comparison of the superconducting transition for one of the Bi-2212 samples as measured by the resistivity, $d \rho / d T$ and $\chi_{\sigma}^{-1}$. As can be seen in Fig. 1(c), the $\chi_{\sigma}^{-1}$ representation clearly reveals the two-stage character of the transition. ${ }^{6}$ Thus, we can readily identify the temperature interval relevant for studying fluctuations in the normal phase, and the regime dominated by mesoscopic granularity close to the $R=0$ state. The temperature crossover $T_{p}$, which corresponds to the maximum of $d \rho / d T$, delimits approximately the two distinct resistive regimes. The two-stage character of the transition is not as easily perceived in the resistivity vs temperature plots of Fig. $1(\mathrm{a})$.

\section{RESULTS}

\section{A. Bi-2212}

Results shown in Fig. 1 for sample B2-a of the Bi-2212 system are representative of the essential characteristics of the fluctuation conductivity in our samples. Figure 1(c) shows that above, but close to $T_{c}$, the variation of $1 / \chi_{\sigma}$ as a function of temperature may be fitted to two straight lines. These fits are labeled by the indices $\lambda_{G}$ and $\lambda_{\mathrm{cr}}$, denoted on the picture. Farther from $T_{p}$, the regime corresponding to the exponent $\lambda_{G} \cong 0.5$ is quite robust with respect to current variation or heat treatment of the samples, and is interpreted as resulting from Gaussian fluctuations in the microscopically granular array. Closer to $T_{p}$, a crossover is observed to another power-law behavior, in which the exponent's value, $\lambda_{\mathrm{cr}} \cong 3.0$, is characteristic of critical conductivity fluctuations in classical granular superconductors. ${ }^{5,7}$ An expanded view of the data in the $\lambda_{\text {cr }}$ regime is shown in Fig. 2(a).

Below $T_{p}$, and near the zero resistance state, where effects related to mesoscopic granularity are supposed to prevail, the fluctuation conductivity is also described by a power law of the type

$$
\Delta \sigma \sim\left(T-T_{\mathrm{co}}\right)^{-s},
$$

where $T_{\text {co }}$ is a temperature dependent on the applied current and magnetic field which is close to the so-called zero resis-

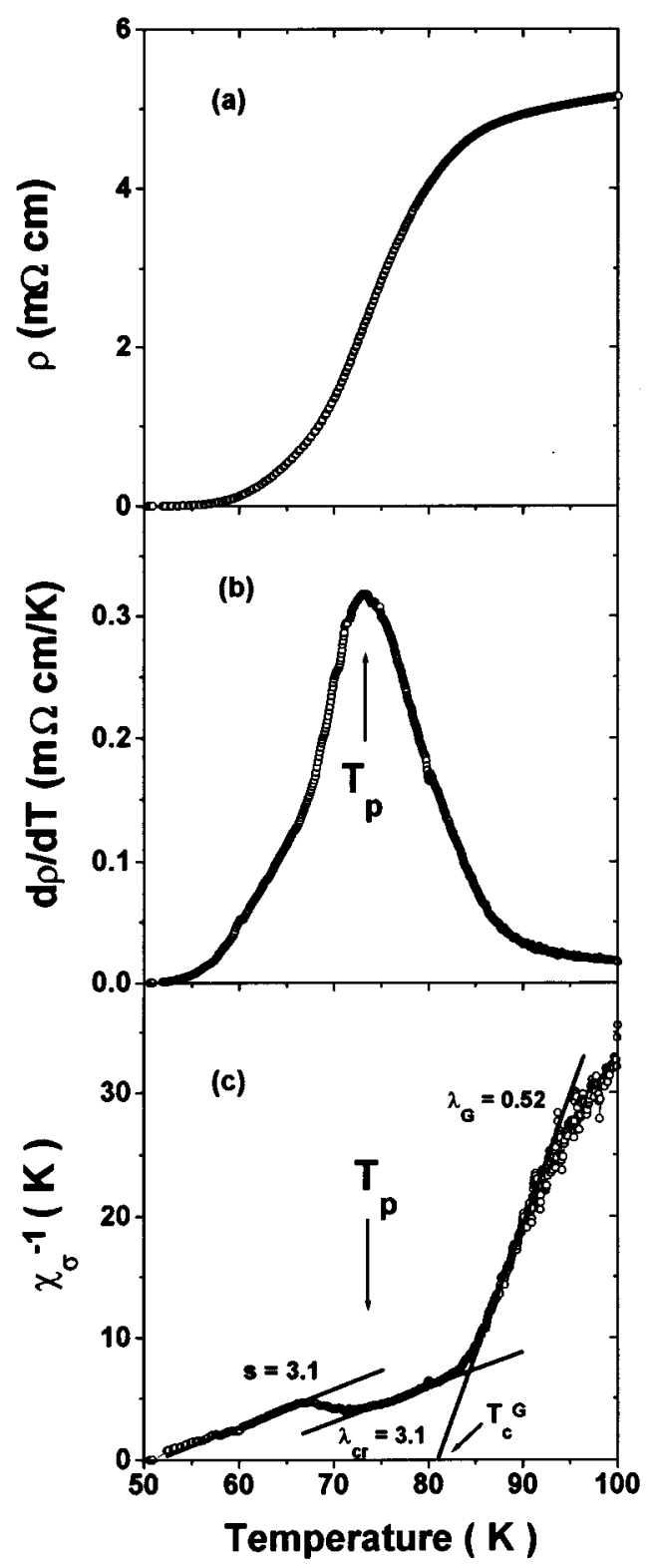

FIG. 1. Representative results of the conductivity transition in sample B2-a of the Bi-2212 system. Panel (a) is a resistivity vs temperature plot. Panel (b) shows the temperature derivative of the resistivity, and panel (c) shows the inverse of the logarithmic derivative of the conductivity as a function of $T$, in the same temperature interval as in panels (a) and (b). Straight lines are fits to Eq. (4) and the corresponding exponents are quoted. Also signaled are the temperatures $T_{p}$ of the maximum of $d \rho / d T$ and $T_{c}^{G}$, which is obtained from extrapolation of the Gaussian power-law behavior (see text).

tance temperature. Indeed, for results in Fig. 1 we have found $s \cong 3$, which is the expected value for critical conductivity fluctuations in granular arrays. ${ }^{7,9}$ The two parallel straight lines in Fig. 1(c) stress the similar critical behaviors of the $s$ and $\lambda_{\text {cr }}$ regimes. The $s$ regime is observed independently of the current of measurement. However for current densities in excess of $100 \mathrm{~mA} / \mathrm{cm}^{2}$, a crossover is observed to another power-law regime with exponent $s^{\prime} \cong 4$ when the temperature approaches $T_{\text {co }}$. Such crossover was first noticed in ceramic $\mathrm{YBa}_{2} \mathrm{Cu}_{3} \mathrm{O}_{7-\delta}$ samples. ${ }^{16}$ In Fig. 2(b) we show an expanded view of $\chi_{\sigma}^{-1}$ close to the $R=0$ state for 


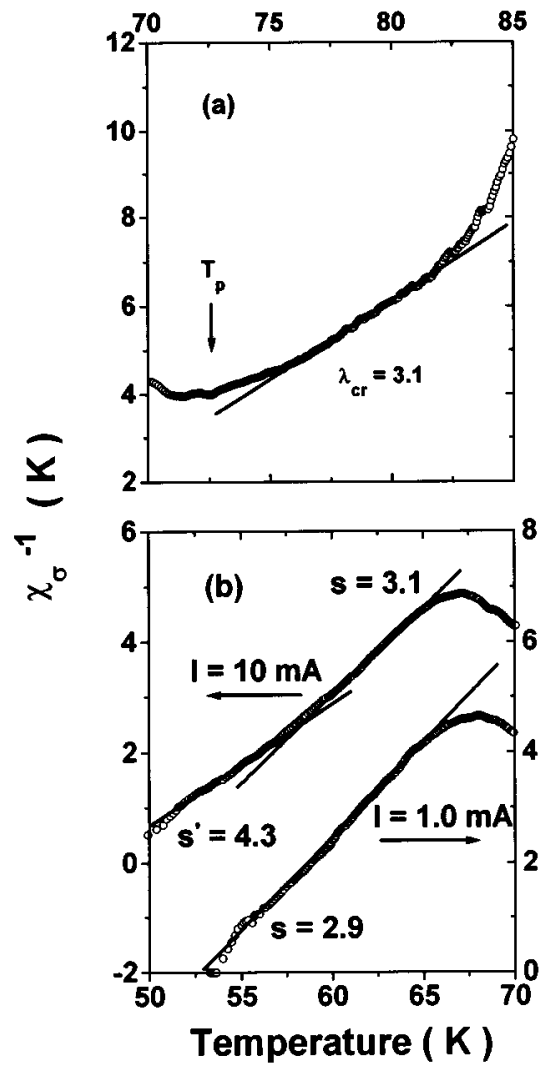

FIG. 2. Expanded views of the inverse of the logarithmic temperature derivative of the conductivity $\chi_{\sigma}^{-1}$ for the Bi-2212 system (sample B2-a). (a) Critical regime, with exponent $\lambda_{\text {cr }} \cong 3$. (b) Results close to the $R=0$ state obtained in $j=32 \mathrm{~mA} / \mathrm{cm}^{2}$ ( $I$ $=1.0 \mathrm{~mA})$ and $j=320 \mathrm{~mA} / \mathrm{cm}^{2} \quad(I=10 \mathrm{~mA})$. Exponents are quoted.

two measuring currents. For $j=32 \mathrm{~mA} / \mathrm{cm}^{2}$ we observe only the regime corresponding to $s \cong 3$, whereas for $j$ $=320 \mathrm{~mA} / \mathrm{cm}^{2}$ a crossover can be identified to $s^{\prime} \cong 4$ when the temperature decreases towards the $R=0$ state.

Another of the Bi-2212 samples, namely B2-b, presents essentially the same behavior as B2-a, except that we could not observe the granular $s^{\prime}$ regime, as the measuring currents were kept very low in this case. The third $\mathrm{Bi}-2212$ sample (B2-c) was submitted to an annealing at $650{ }^{\circ} \mathrm{C}$. This probably enhances the quality of the grains, as may be inferred from the increase in $T_{p}$ (see Table I) and from the nonobservance of the microscopic disorder-induced $\lambda_{\text {cr }}$ regime. On the other hand, the effects of mesoscopic granularity are present and both exponents $s$ and $s^{\prime}$ could be observed. As for the B2-a sample, the $s^{\prime}$ regime was identifiable when the current densities were higher than $100 \mathrm{~mA} / \mathrm{cm}^{2}$.

\section{B. $\mathrm{Bi}-2223$}

Fluctuation conductivity results in the $\mathrm{Bi}-2223$ samples are very similar to those for the Bi-2212 system. In one of the samples, namely, B3-I, we have applied small magnetic fields parallel to the measuring current. Several fields from 1 $\mathrm{G}$ up to $400 \mathrm{G}$ were applied. Figure 3 shows $\chi_{\sigma}^{-1}$ measurements obtained at $H=5.5 \mathrm{G}$. These are representative of the results in the limit of very low fields. Far above $T_{p}$, the

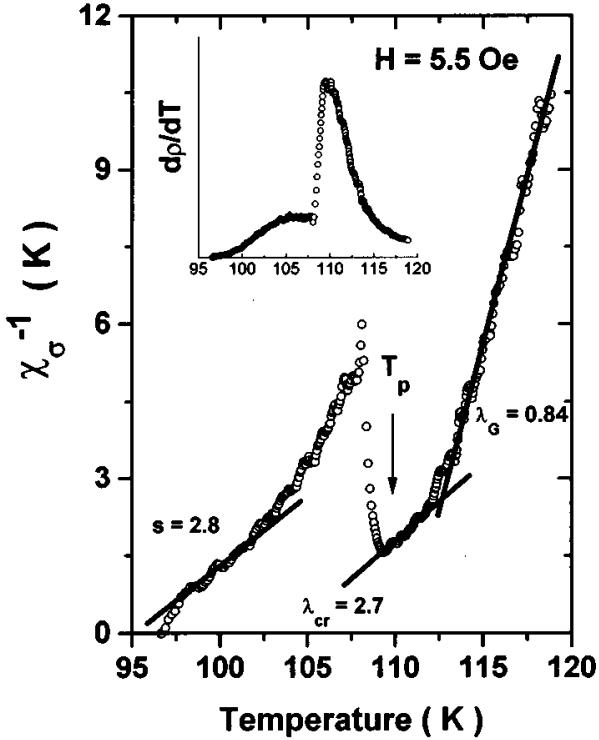

FIG. 3. $\chi_{\sigma}^{-1}$ measurements obtained at $H=5.5 \mathrm{G}$ for the B3-I sample of the $\mathrm{Bi}-2223$ system. In the inset it is shown $d \rho / d T$ for this sample at the same field.

regime corresponding to the exponent $\lambda_{G}=0.84 \pm 0.13$ is interpreted as resulting from almost two-dimensional (2D) Gaussian fluctuations. This behavior is unaffected by the field up to the highest applied value. Closer to $T_{p}$ a crossover to the $\lambda_{\text {cr }}$ regime is clearly observed. This picture is stable, however, only in fields up to $6 \mathrm{G}$. Above, the power law corresponding to the $\lambda_{\text {cr }}$ regime is no longer discernible and $\chi_{\sigma}^{-1}$ becomes visibly rounded in the vicinity of $T_{p}$. Below $T_{p}$, the $s$ regime is also found to be quite unstable against the field. Above $H=20 \mathrm{G}, \chi_{\sigma}^{-1}$ becomes rounded and no power law could be identified in the temperature interval close to the zero resistance state. Also noticeable is the shape of $d \rho / d T$ at low applied fields for sample B3-I. In the inset of Fig. 3, one can observe a steep transition denoting the frontier between intragranular and intergranular resistive behaviors.

Sample B3-II was measured in current densities ranging from 3 to $790 \mathrm{~mA} / \mathrm{cm}^{2}$. Results are current independent and $\chi_{\sigma}^{-1}$ looks very similar to observations in sample B3-I in the limit of small applied fields. The as-sintered B3-III sample was submitted to an annealing of $3 \mathrm{~h}$ in $650{ }^{\circ} \mathrm{C}$ before the resistivity measurements were taken. In this case, the regimes above $T_{p}$ are the same as observed in samples B3-I and B3-II. However, below $T_{p}$ an extended straight line corresponding to $s^{\prime} \cong 4$ could be fitted to the $\chi_{\sigma}^{-1}$ results, as shown in Fig. 4. The inset of this figure allows one to appreciate in more details the $\lambda_{\text {cr }}$ regime.

Sample B3-IV was submitted to an annealing of $12 \mathrm{~h}$ in $650{ }^{\circ} \mathrm{C}$. In this case, as also observed in the Bi-2212 system, the $\lambda_{\text {cr }}$ region is absent. Moreover, the Gaussian exponent decreases to $\lambda_{G} \cong 0.5$. Below $T_{p}$, the heat treatment induces a huge increase of the region dominated by mesoscopic granularity, but the exponent $s \cong 3$ could be identified only for currents below $20 \mathrm{~mA} / \mathrm{cm}^{2}$. Figure 5 illustrate the data for this sample. Table I summarizes the obtained exponents concerning fluctuation conductivity in our Bi-2212 and Bi2223 samples. 


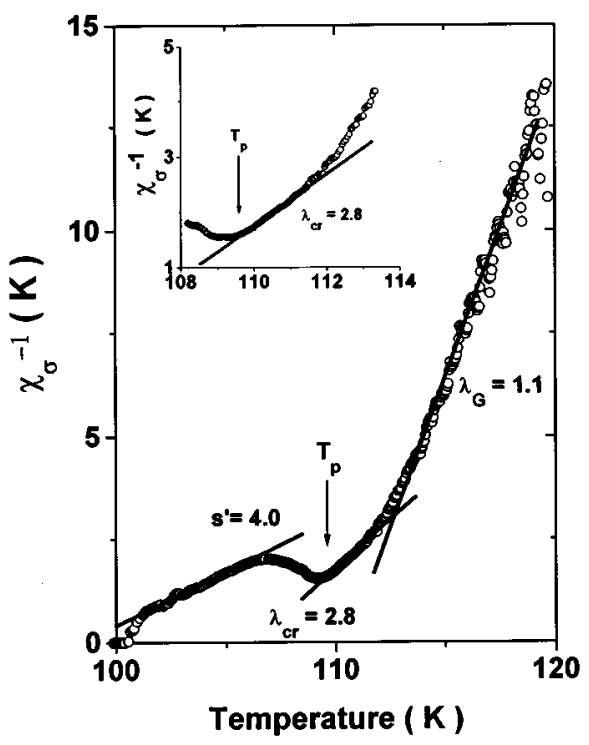

FIG. 4. $\chi_{\sigma}^{-1}$ results for sample B3-III of the Bi-2223 system. In the inset, an enlarged view of the $\lambda_{\mathrm{cr}}$ regime is shown.

\section{DISCUSSION}

Figures 1-5 and Table I show the general characterization of the resistive transition in our $\mathrm{Bi}-2212$ and $\mathrm{Bi}-2223$ ceramic samples. Although strongly disordered at microscopic and mesoscopic levels, the rounded shape of the transition is related to thermal fluctuation effects.

The resistive transition may be roughly described as a two-stage process. The fluctuation conductivity in the normal phase is identified by two power-law regimes in the reduced temperature. Farther from the bulk superconducting transition, which occurs close to $T_{p}$, the obtained exponents correspond to $3 \mathrm{D}$ or $2 \mathrm{D}$ Gaussian fluctuations, depending on the sample. Closer to $T_{p}$, results show the systematic dominance of a critical regime in which the value of the exponent is strongly affected by microscopic inhomogeneities. Below $T_{p}$, in the temperature interval characterizing the approach to the zero resistance state, fluctuation conductivity clearly shows the critical behavior expected for mesoscopic granular superconductors.

\section{A. Fluctuations in the normal phase}

\section{Gaussian regime}

For the Bi-2212 samples, we have observed that the reduced temperature interval where Gaussian fluctuations prevail is limited to $0.06<\varepsilon_{G}<0.14$, where $\varepsilon_{G}=\left(T-T_{c}^{G}\right) / T_{c}^{G}$ is referred to the extrapolated $T_{c}^{G}$, as shown in Fig. 1(c). The Gaussian exponent found for these samples is $\lambda_{G}=0.5$ (see Table I). According to the Aslamazov-Larkin theory, ${ }^{10}$ the Gaussian exponent is given by $\lambda_{G}=2-d / 2$, and a value $\lambda_{G}=0.5$ would be expected for a three-dimensional $(d=3)$ fluctuating system. Our result is quite surprising since $\mathrm{Bi}$ 2212 is characterized by a strong planar anisotropy, and one would expect that the dominant fluctuation regime in the Gaussian region should be typically 2D, as obtained in single-crystal samples. ${ }^{11}$

The theoretical amplitude of fluctuation conductivity in a 3D system is $A=e^{2} / 32 \hbar \xi_{G}(0),{ }^{10}$ where $\xi_{G}(0)$ is the coher-

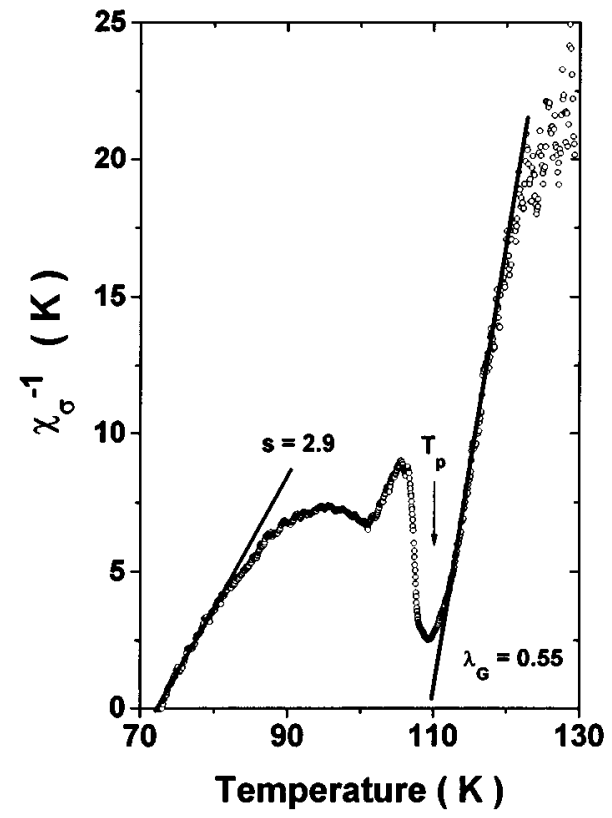

FIG. 5. $\chi_{\sigma}^{-1}$ results for sample B3-IV of the Bi-2223 system. The scaling corresponding to the critical regime dominated by microscopic granularity is not visible in this case.

ence length at zero temperature. The experimental amplitude may be obtained from our results by fitting the fluctuation conductivity data to Eq. (1) in the temperature interval where the Gaussian regimes were identified from the $\chi_{\sigma}^{-1}$ analysis. From the obtained values for $A$ we calculate $\xi_{G}(0) \cong 10 \mathrm{~nm}$ for the B2-a sample and $\xi_{G}(0) \cong 20 \mathrm{~nm}$ for samples B2-b and B2-c. These values are much larger than the intrinsic coherence length of the $\mathrm{Bi}-2212$ superconductor $^{12}$ and are related to the granular nature of the sample. The experimental amplitudes $A$ certainly give overestimated values for $\xi_{G}(0)$ because of the porosity of the ceramic samples, the effect of grain boundary barriers, and the anisotropy of the randomly distributed crystallites. Nevertheless, $\xi_{G}(0)$ may be roughly visualized as an upper limit for a sampledependent correlation length of the microscopically disordered superconductor. In other terms, $\xi_{G}(0)$ would give an upper limit for the size of regions having homogeneous $T_{c}$. Most likely, the mean size of these regions in our samples is considerably smaller than the calculated $\xi_{G}(0)$.

For the Bi-2223, the Gaussian regimes extend from $\varepsilon_{G}$ $=0.03$ to $\varepsilon_{G}=0.10$. For three of the samples, the values of the exponents are consistent with a $2 \mathrm{D}$ or quasi-2D fluctuation spectrum. For sample B3-IV, which was annealed at $650{ }^{\circ} \mathrm{C}$, we have found $\lambda_{G} \cong 0.5$. Again, for the Bi-2223 ceramics, the Gaussian regimes are likely to be describing the fluctuation conductivity of a microscopically granular superconductor. The 2D or quasi-2D exponents found in samples B3-I, B3-II, and B3-III are in good agreement with results previously obtained by Ausloos and co-workers ${ }^{13}$ in a polycrystalline sample of $\mathrm{Bi}-2223$. These authors proposed that a surfacelike percolation backbone underlies fluctuation phenomena in such systems. This model is consistent with the formation process of the $\mathrm{Bi}-2223$ phase, which grows on the surface of $\mathrm{Bi}-2212$ platelets. ${ }^{14}$ The exponent $\lambda_{G} \cong 0.85$, found in samples B3-I and B3-II, is close to the one found by 
Ausloos and co-workers and implies that the fluctuation network develops in a fractal space with spectral dimensionality $\widetilde{d} \cong 2.3 \cong 1+\frac{4}{3}$, where $\frac{4}{3}$ is the well-known fraction dimension for percolation clusters. ${ }^{15} \mathrm{We}$ also note that quasi-2D fluctuation behavior was identified in some $\mathrm{YBa}_{2} \mathrm{Cu}_{3} \mathrm{O}_{7-\delta}$ ceramic samples. ${ }^{6}$ The $3 \mathrm{D}$ regime observed in samples B3-IV is a consequence of the heat treatment at $650{ }^{\circ} \mathrm{C}$, which probably produces a more uniform distribution of the off-stoichiometric oxygen within the crystallites. For this sample, we calculate $\xi_{G}(0) \cong 25 \mathrm{~nm}$, which is of the same order as that obtained in Bi-2212.

\section{Critical fluctuations}

For the most disordered samples, when the temperature is decreased below the Gaussian interval, a crossover is systematically observed in the fluctuation conductivity to a power-law regime described by the exponent $\lambda_{\mathrm{cr}} \cong 3$. We interpret this $\lambda_{\mathrm{cr}}$ regime as being originated from genuine critical fluctuations. The exponent's value is suggestive of granularity. ${ }^{5}$ However, since the characteristic temperature interval occurs in the normal phase, we cannot associate it with classical granularity, typical of samples formed by small superconducting regions which are weak-linked to each other by Josephson couplings. Instead, the $\lambda_{\text {cr }}$ regime in our samples is a clear manifestation of microscopic granularity.

In addition to mesoscopic disorder characteristic of ceramics, our samples are also strongly disordered at a microscopic level. Deviations from the nominal oxygen stoichiometry, $\mathrm{Bi}-\mathrm{Pb}$ interchange, distortions of $\mathrm{Cu}-\mathrm{O}$ polyhedra and polymorphism are among the most frequent microscopic defects in the Bi-based superconductors. ${ }^{16}$ Due to the smallness of the intrinsic coherence length, these deviations from the ideal structure manifest themselves through spatial fluctuations of the critical temperature. The analysis of the Gaussian regimes indicates that $T_{c}(\mathbf{r})$ fluctuates in scales of approximately 10 to $25 \mathrm{~nm}$, which are one order of magnitude larger than the intrinsic coherence length, but are much smaller than the size of crystallites. This situation meets the most important requirement to characterize a microscopically granular state, ${ }^{3}$ where critical behavior is equivalent to that of classical granular systems.

As for to the $3 \mathrm{D}-X Y$ universality class, to which superconductors are supposed to belong, disorder is expected to be relevant. Then, the critical exponents should differ from those predicted ${ }^{16}$ and observed ${ }^{6,10}$ in the homogeneous case. In the critical region, the dynamical scaling theory ${ }^{17,18}$ predicts that the fluctuation conductivity diverges at $T_{c}$ as

$$
\Delta \sigma \sim \varepsilon^{-\nu(2+z-d+\eta)},
$$

where $\varepsilon=\left(T-T_{c}\right) / T_{c}, \nu$ is the coherence length critical exponent, $z$ is the dynamical exponent and $\eta$ takes into account the deviation from the Ornstein-Zernike behavior in the order parameter correlation function. For granular superconductors, it has been demonstrated that $\nu \cong \frac{4}{3} .3,5$ For $d=3$ and assuming that $\eta \cong 0$, the value $\lambda_{\text {cr }} \cong 3.0$ would correspond to an anomalous relaxation with exponent $z \cong 3$.2. This value is intermediate between the homogeneous mean field $z=2$ and the value expected for a spin glass or a dirty ferromagnet system, where $z \cong 4 .{ }^{19}$

\section{B. Fluctuations near the zero resistance state}

From an empirical point of view, the simplest description of our fluctuation conductivity results in the regime approaching the zero resistance state is given by Eq. (5). The power-law behavior, which holds for low currents and low applied magnetic fields, is indicative of the occurrence of a paracoherent-coherent transition, ${ }^{5,6}$ characteristic of a granular superconducting network. At $T_{\mathrm{co}}$, the fluctuating phases of the order parameter in each mesoscopic grain become long-range ordered as a consequence of a thermally controlled percolationlike process that activates weak links between grains. The obtained exponent, $s \cong 3$, is known to characterize the resistive transition of artificially prepared granular arrays consisting of small superconducting particles embedded in an insulating matrix..$^{5}$ The fluctuation conductivity of ceramic $\mathrm{YBa}_{2} \mathrm{Cu}_{3} \mathrm{O}_{7-\delta}$ near the zero resistance state is also known to be described by Eq. (5) with exponent close to 3 . $^{6,7}$

As exemplified in Fig. 2 and shown in Table I, in some experiments, performed with relatively high current densities, a crossover in $\Delta \sigma$ to a power-law behavior with exponent $s^{\prime} \cong 4$ is observed when the temperature approaches the $R=0$ point. In one of the Bi-2223 samples, namely, sample B3-III, the $s^{\prime} \cong 4$ is the only scaling regime observed below $T_{c}$, as shown in Fig. 4. If attributable to a critical phenomenon, and accordingly to Eq. (6) where $\nu$ is kept equal to $\frac{4}{3}$, the exponent $s^{\prime} \cong 4$ would correspond to $z \cong 4$. This scenario fits well into a description based on a vortex-glass transition. ${ }^{20}$ The vortex-glass transition with exponents close to ours have been observed in epitaxial films ${ }^{21}$ or single crystals $^{22}$ by resistivity and nonlinear I-V measurements at relatively high applied fields. In agreement with the analysis presented in Ref. 21, we only observe true critical vortex glass fluctuations above $T_{\text {co }}$ and no crossover to vortex-glass Gaussian behavior ${ }^{23}$ could be identified. The dominance of the glass thermodynamics in the limit of small self-fields, in contrast to conventional flux creep descriptions,${ }^{24}$ is probably due to the validity of the phase glass model ${ }^{9,25}$ in the case of strongly disordered ceramic materials. This model represents a dirty limit of the more general vortex-glass theory and its properties are related to Josephson weak-link effects. It is worthwhile to note that, starting from the phase glass Hamiltonian and using a mean-field approach based on the Kubo formula, the author in Ref. 9 found theoretically that fluctuation conductivity is expected to diverge with the exponent $s=3$. This reinforces the interpretation of this regime, as well as for the $s^{\prime} \cong 4$ one, as being intrinsically related to superconducting granularity. As an additional proof, one could also notice that a vortex-glass type of dynamics was observed in transport and magnetic measurements at low fields in ceramic $\mathrm{YBa}_{2} \mathrm{Cu}_{3} \mathrm{O}_{7}{ }^{26}$

\section{CONCLUSIONS}

We studied experimentally the fluctuation conductivity close to $T_{c}$ of disordered samples of the Bi-2212 and Bi2223 superconductors. For the analysis of the data we devised a method based on the determination of the logarithmic derivative of the conductivity with respect to the temperature. The results show clearly that the resistive transition is a two-stage process, and the fluctuation regimes dominating 
each stage could be analyzed separately.

In the normal phase, we identified the occurrence of a Gaussian and a critical scaling regime. Both are dependent on the state of microscopic disorder in the sample. In the Bi-2212 samples, the Gaussian interval is rather extended and correspond to a 3D fluctuation spectrum. In the Bi-2223 samples, the Gaussian regime is consistent with 2D or quasi-2D fluctuations, but may become 3D depending on a heat treatment that modifies the state of microscopic disorder of the sample. Because of the strong planar anisotropy of the Bi-2212 and $\mathrm{Bi}-2223$ superconductors, one could expect that intracrystallite 2D Gaussian fluctuations would dominate in these systems. Our results imply that the strong microscopic disorder modifies the fluctuation spectrum and favors the 3D behavior in the Gaussian interval, as it does in the genuine critical regime. Closer to $T_{c}$, in both systems, we observed a scaling regime with exponent $\lambda_{\mathrm{cr}} \cong 3$ that is typical of critical fluctuations in the conductivity of granular superconductors. This $\lambda_{\text {cr }}$ regime is extremely sensible to applied magnetic fields. We propose that both Gaussian and critical regimes are characteristic of a microscopic granular superconducting state in which the correlation length is larger than the intrinsic coherence length, but much smaller than the typical grain size. The microscopic granularity is a consequence of local disorder that induces spatial fluctuations in $T_{c}(\mathbf{r})$, according to the model proposed in Ref. 3. We note that our interpretation, which attributes to thermal fluctuations the enhancement of the conductivity in the normal phase of our samples, is opposed to the common belief that disorder merely produces noncritical roundings of the transition.
Below the bulk transition, classical granularity effects related to disorder at mesoscopic level dominate the regime describing the approach to the zero resistance state. The fluctuation conductivity diverges with the exponent $s \cong 3$, as expected for granular networks. A vortex-glass exponent $s^{\prime}$ $\cong 4$, which is also related to granularity, is identified in some measurements with relatively high current densities. As generally observed in granular superconductors, the scaling behavior of the conductivity below $T_{c}$ in our samples is strongly dependent on the current of measurement and the applied magnetic field, and is modified or destroyed when current or field exceed some relatively low threshold value. On the other hand, the hierarchical character of the granularity in our samples is additional evidence to some earlier observations ${ }^{6,13,27}$ that the fluctuation spectra of the disordered cuprate superconductors should be defined in a selfsimilar space.

\section{ACKNOWLEDGMENTS}

This work was partially financed by the Brazilian Ministry of Science and Technology under PRONEX/FINEP/ CNPq Contract No. 41.96.0907.00. Partial support was also given by the Fundação de Amparo à Pesquisa do Estado do Rio Grande do Sul (FAPERGS) and the Brazil-France agreement CAPES-COFECUB Contract No. 113/90. We also acknowledge S. A. Sergeenkov for stimulating discussions and a critical reading of the manuscript.
*Author to whom correspondence should be sent; Fax: +55 51 3191762; electronic address: PPUREUR@IF.UFRGS.BR

${ }^{1}$ F. Sharifi, J. Giapintzakis, D. M. Ginsberg, and D. J. Van Harlingen, Physica C 161, 555 (1989).

${ }^{2}$ W. Lang, Physica C 226, 267 (1994).

${ }^{3}$ A. Raboutou, P. Peyral, C. Lebeau, J. Rosenblatt, J. P. Burin, and Y. Fouad, Physica A 207, 271 (1994).

${ }^{4}$ Y. Fouad, A. Raboutou, P. Peyral, C. Lebeau, J. Rosenblatt, J. P. Burin, J. Dehmej, M. Mokhtari, C. Perrin, and M. Sergent, Physica C 235-240, 2841 (1994).

${ }^{5}$ J. Rosenblatt, in Percolation, Localization and Superconductivity, Vol. 109 of NATO Advanced Study Institute, Series B-Physics, edited by A. M. Goldman and S. A. Wolf (Plenum, New York, 1984), p. 431.

${ }^{6}$ P. Pureur, R. Menegotto Costa, P. Rodrigues, Jr., J. Schaf, and J. V. Kunzler, Phys. Rev. B 47, 11420 (1993).

${ }^{7}$ P. Peyral, C. Lebeau, J. Rosenblatt, A. Raboutou, C. Perrin, O. Pena, and M. Sergent, J. Less-Common Met. 151, 49 (1989).

${ }^{8}$ Y. Idemoto, S. Ichikawa, and K. Fueki, Physica C 181, 171 (1991).

${ }^{9}$ S. A. Sergeenkov, Z. Phys. B 82, 325 (1991).

${ }^{10}$ L. G. Aslamazov and A. I. Larkin, Fiz. Tverd. Tela (Leningrad) 10, 1104 (1968) [ Sov. Phys. Solid State 10, 875 (1968)].

${ }^{11}$ A. K. Pradhan, S. B. Roy, P. Chaddah, C. Chen, and B. M. Wanklyn, Phys. Rev. B 50, 7180 (1994); P. Pureur, R. Menegotto Costa, P. Rogrigues, Jr., J. V. Kunzler, J. Schaf, L. Ghivelder, J. A. Campá, and I. Rasines, Physica C 235-240, 1939 (1994).

${ }^{12}$ T. T. M. Palstra, B. Batlogg, L. F. Schneemeyer, R. B. van Dover, and J. V. Waszczak, Phys. Rev. B 38, 5102 (1988).

${ }^{13}$ M. Ausloos, P. Clippe, and Ch. Laurent, Phys. Rev. B 41, 9506 (1990).

${ }^{14}$ M. T. Malachevsky and D. A. Esparza, Thermochim. Acta 265, 1997 (1995).

${ }^{15}$ S. Alexander and R. Orbach, J. Phys. (France) Lett. 43, L625 (1982).

${ }^{16}$ O. Eibl, Physica C 168, 215 (1990).

${ }^{17}$ C. J. Lobb, Phys. Rev. B 36, 3930 (1987).

${ }^{18}$ P. C. Hohenberg and B. I. Halperin, Rev. Mod. Phys. 49, 435 (1977).

${ }^{19}$ K. Binder and A. D. Young, Rev. Mod. Phys. 58, 801 (1986).

${ }^{20}$ D. S. Fischer, M. P. A. Fischer, and D. A. Huse, Phys. Rev. B 43, 130 (1991).

${ }^{21}$ M. Friesen, J. Deak, L. Hou, and M. McElfresh, Phys. Rev. B 54, 3525 (1996).

${ }^{22}$ H. Safar, P. L. Gammel, D. J. Bishop, D. B. Mitzi, and A. Kapitulnik, Phys. Rev. Lett. 68, 2672 (1992).

${ }^{23}$ A. T. Dorsey, M. Huang, and M. P. A. Fisher, Phys. Rev. B 45, 523 (1992).

${ }^{24}$ T. T. M. Palstra, B. Batlogg, R. B. van Dover, L. F. Schneemeyer, and J. V. Waszczak, Phys. Rev. B 41, 6621 (1990).

${ }^{25}$ D. A. Huse and H. S. Seung, Phys. Rev. B 42, 1059 (1990).

${ }^{26} \mathrm{~S}$. Li, M. Fistul, J. Deak, P. Metcalf, and M. McElfresh, Phys. Rev. B 52, R747 (1995).

${ }^{27}$ N. Mori, J. A. Wilson, and H. Ozaki, Phys. Rev. B 45, 10633 (1992). 